\title{
Static postural sway, proprioception, and maximal voluntary quadriceps contraction in patients with knee osteoarthritis and normal control subjects
}

\author{
B S Hassan, S Mockett, M Doherty
}

\begin{abstract}
Objectives-To investigate whether subjects with knee osteoarthritis (OA) have reduced static postural control, knee proprioceptive acuity, and maximal voluntary contraction (MVC) of the quadriceps compared with normal controls, and to determine possible independent predictors of static postural sway.

Methods-77 subjects with symptomatic and radiographic knee OA (58 women, 19 men; mean age 63.4 years, range $36-82$ ) and 63 controls with asymptomatic and clinically normal knees (45 women, 18 men; mean age 63 years, range 46-85) underwent assessment of static postural sway. 108 subjects (59 patients, 49 controls) also underwent assessment of knee proprioceptive activity and MVC (including calculation of quadriceps activation). In patients with knee $O A$ knee pain, stiffness, and functional disability were assessed using the WOMAC Index. The height $(\mathrm{m})$ and weight $(\mathrm{kg})$ of all subjects was assessed.
\end{abstract}

Results-Compared with controls, patients with knee OA were heavier (mean difference $15.3 \mathrm{~kg}, \mathrm{p}<0.001$ ), had increased postural lateral sway (controls: median 2.3, interquartile (IQ) range 1.8-2.9; patients: median 4.7 , IQ range 1.9-4.7, p<0.001), reduced proprioceptive acuity (controls: mean 7.9 , 95\% CI 6.9 to 8.9 ; patients: mean $12.0,95 \%$ CI 10.5 to 13.6 , p<0.001), weaker quadriceps strength (controls: mean 22.5 , $95 \%$ CI 19.9 to 24.6 ; patients: mean $14.7,95 \%$ CI 12.5 to 16.9 , p <0.001), and less percentage activation of quadriceps (controls: mean $87.4,95 \%$ CI 80.7 to 94.2 ; patients: mean $66.0,95 \%$ CI 58.8 to $73.2, p<0.001)$. The significant predictors of postural sway were knee pain and the ratio of $M V C /$ body weight.

Conclusions-Compared with age and sex matched controls, subjects with symptomatic knee OA have quadriceps weakness, reduced knee proprioception, and increased postural sway. Pain and muscle strength may particularly influence postural sway. The interaction between physiological, structural, and functional abnormalities in knee $\mathrm{OA}$ deserves further study.

(Ann Rheum Dis 2001;60:612-618)

of Nottingham

S Mockett

Correspondence to:

Professor Doherty

Michael.Doherty@Nottingham. ac.uk

Accepted 15 November 2000
Control of balance is vital to everyday life. It allows performance of activities that range from maintenance of static positions to complex dynamic activities. Postural control is complex and on the afferent side involves the central processing of peripheral sensory input from vestibular, visual, and proprioceptive pathways, whereas the efferent side involves the precise recruitment of specific (and varying) populations of motor units. Peripheral proprioception involves various sensory receptors, including cutaneous touch and pressure receptors, mechanoreceptors of synovial joints, muscle spindles, and tendon Golgi organs. Muscle spindles are the more important for detecting changes in joint angulation in the mid-range of movement, whereas joint mechanoreceptors (pacinian corpuscles and Ruffini's end organs), which detect stretch of ligaments and deep tissues, are more important at the extremes of joint movement. The knee joint has a rich sensory innervation and has mechanoreceptors in the anterior and posterior cruciate ligaments, collateral ligaments, and menisci. ${ }^{1-4}$

Osteoarthritis (OA) may result in changes that affect not only intracapsular tissues but also periarticular tissues, such as ligaments, capsule, tendons, and muscle. ${ }^{56}$ Subjects with knee OA are known to have impaired proprioception compared with age matched controls, ${ }^{78}$ and histology of ligaments from OA knees shows marked reduction in the number of mechanoreceptors. Knee OA is also associated with a $50-60 \%$ reduction in maximum quadriceps torque, ${ }^{9}$ possibly resulting from disuse atrophy and arthrogenic inhibition. ${ }^{10}$ Electromyographic studies show recruitment of more muscle fibres in subjects with painful knee OA than in normal controls for similar tasks such as standing, as well as unequal activation of the individual muscle groups of the quadriceps femoris. Such unequal activation may result from muscle insufficiency, neuromuscular incoordination, or pain. ${ }^{11}$

Postural (or balance) control can be assessed under either static or dynamic conditions. ${ }^{12}{ }^{13}$ Dynamic tests assess balance control during voluntary execution of a movement, such as walking or rising from a chair. Static tests assess the ability to maintain an upright position in various situations, such as with the eyes closed or with expected or unexpected perturbance. The tests measure the frequency against time of postural sway by assessing medial-lateral displacement of the centre of pressure. ${ }^{12} 13$ Large excursions of the centre of pressure are generally assumed to be signs of poor balance.

The primary objective of this study was to investigate whether control of balance as assessed by static postural control is impaired in subjects with knee OA compared with age 
matched controls. The secondary objective was to explore possible independent predictors of increased sway, such as knee pain, knee proprioception, or maximal voluntary contraction (MVC) of the quadriceps.

\section{Methods}

The study was approved by the research ethics committee of Nottingham City Hospital. Informed written consent was obtained from all participants.

STUDY PARTICIPANTS

Patients with knee OA were recruited from the weekly knee OA clinic at the City Hospital, Nottingham. All patients with OA had symptoms and signs consistent with knee $\mathrm{OA}$ and radiographic evidence of definite osteophyte, with definite joint space narrowing affecting at least one knee compartment (medial tibiofemoral or patellofemoral) viewed on standard radiographs (standing extended anteroposterior and skyline $30^{\circ}$ flexion views). No patients had symptoms, signs, or radiographic findings to suggest coexistent inflammatory or other locomotor disease. Non-OA controls were recruited by local advertisement and comprised staff at the City Hospital Nottingham and spouses of patients with knee OA. Controls were included on the basis of (a) no current or past knee pain and no history of major knee trauma, and $(b)$ normal findings on clinical examination of the knees and other target sites for OA (hips, first carpometacarpal joint, distal interphalangeal finger joints). Controls were matched for age and sex to the OA participants. No patient or control was included if they had a history of faints, vertigo, diabetes mellitus, Parkinsonism, or other condition that might impair balance. Subjects were excluded if they were taking drugs for any of the above conditions or drugs with the known side effect of causing any of the above symptoms. Patients were not excluded if they were taking non-steroidal anti-inflammatory drugs.

ASSESSMENTS

As a self perceived assessment of balance, all participants were asked if they had experienced any problems with balance, with "giving way" of their legs, or had had any fall in the past two years. All were weighed $(\mathrm{kg})$ and had their height measured (m). Patients' pain, stiffness, and physical function were assessed. The self administered WOMAC questionnaire was used for this purpose. It is an activity based questionnaire designed for patients with knee and hip OA. Its validity, reliability, and relative efficiency have been tested and it has been shown to be a reliable and valid multidimensional health status instrument. ${ }^{14}$ The questionnaire includes 24 questions (five pain, two stiffness, and 17 physical function disability).

Postural sway

Static postural stability was assessed with the Balance Performance Monitor (BPM) (SMS Technologies, Harlow, Essex CM19 5TL). The machine is designed to assess upright weightbearing status and comprises two foot plates connected to a feedback unit. For each subject the position of the foot plates was adjusted to match their normal stance. They were then asked to stand steadily on the foot plates with their shoes on, arms at their sides, and eyes closed. The procedure was performed twice, the first to acclimatise the subject, the second being used for measurement. Data were acquired at $10 \mathrm{~Hz}$ over a period of 30 seconds, and presented both numerically and graphically. Graphical information presented a trace as the subject's weight shifted in lateral and anteroposterior directions and as a centre of gravity trace indicating the movement of the subject's centre of balance about their base of support during the 30 second test. The BPM data is recorded in arbitrary units on a scale from 0 to 100 , from which it is possible to derive values of weight distribution (balance coefficient) and relative movement (sway coefficient). The balance coefficient is the mean weight that shifts during the 30 second test period in a left-right or lateral direction. The sway coefficient is the standard deviation of the balance coefficient. Greater balance and sway coefficients indicate a greater amount of postural instability. Reproducibility was assessed by repeat measurement of postural sway on two occasions one week apart on 20 subjects (10 patients with knee OA, 10 controls).

\section{Knee joint proprioception}

Proprioceptive acuity was assessed by the ability to reproduce passive positioning of the leg with eyes closed using custom designed apparatus based on published descriptions. ${ }^{715}$ The apparatus comprised a chair with a device that enabled passive positioning of the subject's leg, a protractor with one-degree divisions attached to the side of the chair, a counterbalance to the weight of the subject to ensure that the leg felt weightless during leg movement, and inflatable pneumatic cuffs (Centromed, Ashford, Kent TN24 0SJ) for the thigh and lower leg to eliminate any contribution from cutaneous receptors and to avoid skin contact with clothing. The $90^{\circ}$ flexion of the knee corresponded to $90^{\circ}$, and full extension of the leg to $180^{\circ}$, on the protractor. Each subject was seated on the chair with hips and knees at $90^{\circ}$ flexion. The upper pneumatic cuff was placed around the whole thigh from just above the knee, and the lower cuff around the whole foot and calf from just below the knee. The following procedure was then performed with eyes closed and hands folded across the chest. The leg was moved passively, positioned at a certain position (the criterion angle) and held there for five seconds. The leg was then returned passively by the examiner to the original position and the subject asked to actively reproduce the criterion angle. Criterion angles were randomly selected between $90^{\circ}$ flexion and full extension. The difference between the criterion and reproduced angle was taken as a measure of proprioceptive acuity. The whole procedure was performed four times, the first 
as a trial to familiarise the subject with the procedure. The average of the last three procedures was taken as the proprioceptive acuity for that limb. Reproducibility was assessed by repeat measurement of proprioception on two occasions one week apart on 20 subjects (10 patients with knee OA, 10 controls).

\section{Maximal voluntary contraction and percentage activation of the quadriceps}

Maximal quadriceps strength was measured isometrically using a modified Tornvall chair. Each subject was seated on the chair with hips and knees flexed to $90^{\circ}$. Restraining straps were fitted across the pelvis and upper chest to minimise unwanted pelvic and upper trunk movements. A non-extensile strap was placed just above the medial malleolus, the other end being connected by a metal chain to a strain gauge (TKA load cell Techni Measure, Studley) that was attached to a bar at the back of the chair such that the line of the pull was horizontal. The output from the strain gauge was amplified by an amplifier and a digital read out (Techni Measure, Studley). The final output was read onto a chart recorder (Recorderlab, Surrey SM2 5PS). Each subject was asked to straighten the test leg, pushing as hard as possible against the strap. The procedure was performed three times with a few seconds rest between each. The highest value obtained was regarded as the MVC. The degree of quadriceps activation was estimated by superimposition of an electrical current given through two electrodes consisting of aluminium foil covered in cloth dampened in $0.9 \%$ saline.${ }^{16}$ These were placed on the anterior thigh. Stimulation was achieved with a Digitimer High Voltage Stimulator (DS7-H, Digitimer Ltd, Welwyn Garden City). The device was set to deliver a current of 1 A with stimulation duration of $50 \mathrm{~ms}$. The voltage was adjusted to achieve approximately $20 \%$ quadriceps activation. Once the voltage was set, the subject received a set of three stimulations while at rest (baseline "twitches"). The subject was then asked to perform maximal contractions and to sustain this. Three further currents were delivered with an interval of one second between each. The percentage activation was calculated according to Bigland-Ritchie et $a l .{ }^{17}$ Reproducibility of the instrument for assessment of MVC and quadriceps activation has previously been established in our unit ${ }^{16}:$ the coefficient of variation is $<10 \%$.

\section{STATISTICAL ANALYSIS}

Only data relating to the worst performing leg were analysed. ${ }^{18}$ Statistical analysis was performed using "SPSS" v80. All tests were two tailed. Reproducibility for sway and proprioception was assessed by the method of Bland and Altman. ${ }^{19}$ The intraclass correlation (ICC) and $\alpha$ were also measured. For normally distributed variables data are presented as means and 95\% CI. Differences between these variables were assessed using Student's $t$ test. The non-normally distributed variables are presented as medians and interquartile (IQ) ranges with differences assessed using the Mann-Whitney U test. The degree of association between the measured variables for the whole cohort was estimated using partial (to control for OA) and Spearman's rank correlations for subjects with knee OA.

Multiple regression analysis was used to determine the possible predictors of postural sway in the whole cohort and then in subjects with knee OA. Multiple regression analysis was also used to determine the extent of physical disability (as assessed by the WOMAC) of subjects with knee OA.

Multivariate analysis of variance (MANOVA) was used to analyse the differences in postural sway, proprioception, MVC, and percentage activation of quadriceps, controlling for weight between patients with $\mathrm{OA}$ and controls.

\section{Results}

One hundred and forty subjects were recruited to the study, 77 patients with OA (58 women, 19 men), 12 of these had unilateral knee OA, and 63 controls ( 45 women, 18 men). Table 1 shows the anthropometric data. Patients and controls were similar in age and height, but patients with OA were significantly heavier than controls $(95 \%$ CI of difference -0.60 to $-10.10, p<0.001)$. None of the controls reported any problems with balance, whereas $38(49 \%)$ of the patients with OA had noticed some problem with their balance. This was

Table 1 Anthropometric data, postural sway, proprioceptive acuity (degrees), $M V C^{\star}$ (kgf), quadriceps activation for the controls and patients. The data are presented as means and $95 \%$ CI unless otherwise stated

\begin{tabular}{|c|c|c|c|c|c|c|}
\hline & \multicolumn{2}{|l|}{ Controls } & \multicolumn{2}{|l|}{ Patients } & \multirow{2}{*}{$\begin{array}{l}\text { Mean difference } \\
\text { (95\% CI of difference) }\end{array}$} & \multirow[b]{2}{*}{$p$ Value } \\
\hline & No (\%) & Mean $(95 \%$ CI) & No (\%) & Mean $(95 \%$ CI) & & \\
\hline \multicolumn{7}{|l|}{ Anthropometric data } \\
\hline Male & $18(29)$ & & $19(25)$ & & & \\
\hline Female & $45(71)$ & & $58(75)$ & & & \\
\hline Total & 63 & & 77 & & & \\
\hline Age (years) & & $63(60.1$ to 65.5$)$ & & $63.4(61.0$ to 65.7 & $-0.61(-4.2$ to 3.0$)$ & 0.47 \\
\hline Height (m) & & $1.66(1.64$ to 1.68$)$ & & $1.65(1.63$ to 1.67$)$ & $0.87(-1.95$ to 3.70$)$ & 0.45 \\
\hline Weight (kg) & & $68.7(66.3$ to 71.1$)$ & & $83.4(79.6$ to 87.10$)$ & $-15.34(-20.6$ to -10.10$)$ & $<0.001$ \\
\hline \multicolumn{7}{|c|}{ Postural sway (median, IQ range) } \\
\hline Lateral & & $2.3(1.8-2.9)$ & & $4.7(1.9-4.7)$ & & $<0.001$ \\
\hline Anteroposterior (right) & & $3.3(2.3-5.2)$ & & $4.4(3.4-6.0)$ & & $<0.001$ \\
\hline Anteroposterior (left) & & $3.3(2.2-4.2)$ & & $4.4(3.1-6.4)$ & & $<0.001$ \\
\hline Proprioceptive acuity & 54 & $7.9(6.9$ to 8.9$)$ & 66 & $12.0(10.5$ to 13.6$)$ & $-4.1(-6.1$ to -2.2$)$ & $<0.001$ \\
\hline MVC (kgf) & 49 & $22.5(19.9$ to 24.61$)$ & 59 & $14.7(12.54$ to 16.86$)$ & $7.5(4.40$ to 10.72$)$ & $<0.001$ \\
\hline Quadriceps activation (\%) & 47 & $87.40(80.7$ to 94.20$)$ & 57 & $66.0(58.80$ to 73.20$)$ & $21.4(11.51$ to 31.34$)$ & $<0.001$ \\
\hline MVC/weight & 47 & $0.32(0.30$ to 0.36$)$ & 57 & $0.18(0.15$ to 0.21$)$ & $0.15(0.10$ to 0.20$)$ & $<0.001$ \\
\hline
\end{tabular}

${ }^{\star} \mathrm{MVC}=$ maximal voluntary quadriceps 
Table 2 Scores for pain stiffness and physical function disability for subjects with knee osteoarthritis

\begin{tabular}{lllll}
\hline Subscale of WOMAC & Median & IQ range & Total range & $\begin{array}{l}\text { Maximum } \\
\text { possible score }\end{array}$ \\
\hline Pain & 10 & $7.0-12.0$ & $3.00-16.0$ & 20 \\
Stiffness & 5 & $3.0-6.0$ & $1.0-8.0$ & 8 \\
Physical function disability & 36 & $22.0-42.0$ & $7.0-59.0$ & 68 \\
\hline
\end{tabular}

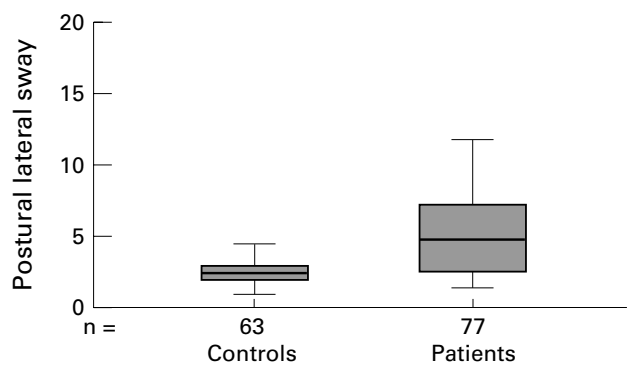

Figure 1 Box plots showing postural lateral sway in controls $(n=63)$ and patients $(n=77)$.

variously described as "feeling wobbly", "tripping readily over things", "swaying", and "feeling unsteady". Of these 38 patients, 23 also reported one or more falls within the previous two years. Postural control was assessed in all 140 subjects. One hundred and twenty subjects (66 patients with OA, 54 controls) were willing to have assessment of proprioception, and 108 of these (59 patients with OA, 49 controls) also agreed to assessment of quadriceps strength and twitch superimposition.

PAIN, STIFFNESS, AND PHYSICAL FUNCTION DISABILITY

Table 2 shows the scores for pain stiffness and physical function disability for subjects with knee OA.

POSTURAL SWAY

For reproducibility the means and the differences of the two assessments on each subject were calculated and plotted on a scatter graph. All data points were within two standard deviations from the mean. That is $95 \%$ of the differences were less than 2SD thus meeting the definition of repeatability of the British Standards Institution. $\alpha$ was 0.87 and the ICCs for the two trials were 0.87 (95\% CI 0.68 to 0.95 ).

Table 1 and figs 1 and 2 show the results for study participants. Subjects with knee OA swayed significantly more than controls in both lateral $(\mathrm{p}<0.001)$ and in anteroposterior

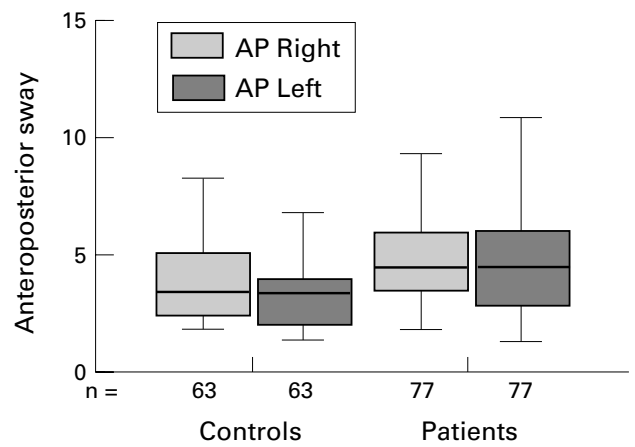

Figure 2 Postural sway in anteroposterior direction on right foot (AP Right) and left foot (AP Left) for controls $(n=63)$, and patients $(n=77)$.

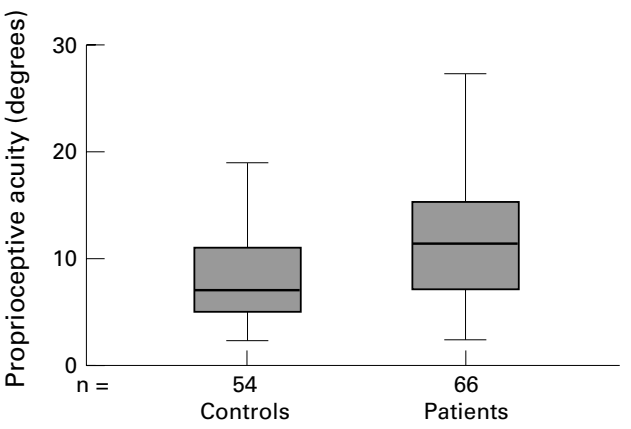

Figure 3 Proprioceptive acuity (degrees) for controls $(n=54)$ and patients $(n=66)$.

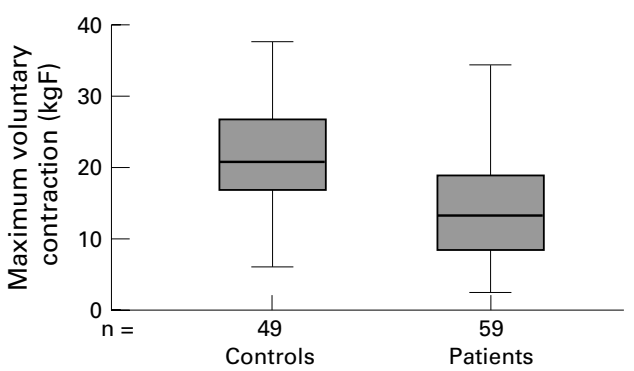

Figure 4 Maximal voluntary contraction for controls $(n=49)$ and patients $(n=59)$.

$(p<0.001)$ directions. Comparison of unilateral and bilateral knee OA showed no significant difference, but with only 12 subjects with unilateral $\mathrm{OA}$, numbers were too small to be conclusive.

PROPRIOCEPTIVE ACUITY

For repeat measures all data points were within two standard deviations from the mean-that is, $95 \%$ of the differences were less than 2SD. $\alpha$ equalled 0.90 and ICCs for the two trials were 0.895 (95\% CI 0.74 to 0.96 ). Table 1 and fig 3 show the results for patients and controls. Patients with OA had a greater mean error than controls - that is, they were less accurate in producing the criterion angle than controls ( $95 \%$ CI of difference -6.10 to -2.20 , $\mathrm{p}<0.001)$.

MVC AND PERCENTAGE ACTIVATION OF THE QUADRICEPS

Table 1 and fig 4 show the data for muscle assessment. Controls exerted significantly more voluntary extensor force than patients with OA (95\% CI of difference 4.40 to 10.72 , $\mathrm{p}<0.001)$. The ratio of $\mathrm{MVC} /$ weight was

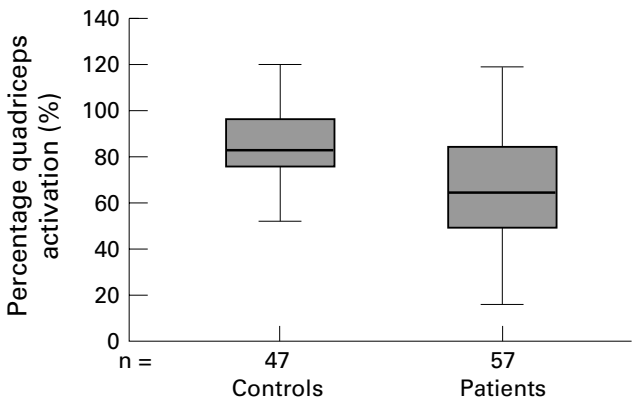

Figure 5 Percentage quadriceps activation for controls $(n=47)$ and patients $(n=57)$. 
Table 3 Regression analyses of postural sway and physical disability (WOMAC score)

\begin{tabular}{|c|c|c|c|c|c|c|}
\hline Dependent variable & $\begin{array}{l}\text { Adjusted } R^{2} \\
\text { (as percentage) }\end{array}$ & Independent variable & $B$ & $\begin{array}{l}\text { Standard } \\
\text { error of } B\end{array}$ & $t$ Value & Significance \\
\hline \multicolumn{7}{|c|}{ (A) All subjects ( $\mathrm{n}=104,47$ controls and 57 patients) } \\
\hline \multirow[t]{4}{*}{ Postural sway } & 47 & Constant & -0.912 & 0.43 & -2.13 & 0.035 \\
\hline & & Knee OA+ & 0.23 & -0.05 & 4.56 & $<0.001$ \\
\hline & & Weight $(\mathrm{kg})$ & 0.004 & 0.002 & 2.80 & 0.005 \\
\hline & & $\mathrm{MVC}^{\star}(\mathrm{kgf})$ & -0.008 & 0.003 & -3.00 & 0.004 \\
\hline \multicolumn{7}{|c|}{ (B) Knee OA + patients only ( $\mathrm{n}=57)$} \\
\hline \multirow[t]{3}{*}{ Postural sway } & 30 & Constant & -0.52 & 0.118 & -0.52 & 0.25 \\
\hline & & MVC/weight $(\mathrm{kgf} / \mathrm{kg})$ & -0.41 & 0.122 & -3.33 & 0.002 \\
\hline & & Pain (WOMAC score) & 0.030 & 0.009 & 2.90 & 0.001 \\
\hline \multicolumn{7}{|c|}{ (C) Knee OA + patients only $(\mathrm{n}=57)$} \\
\hline \multirow[t]{5}{*}{ Physical disability } & 66 & Constant & -10.01 & 9.20 & -1.09 & 0.28 \\
\hline & & MVC (kgf) & -0.26 & 0.112 & -2.28 & 0.027 \\
\hline & & Pain (WOMAC score) & 2.24 & 0.30 & 7.51 & $<0.001$ \\
\hline & & Weight $(\mathrm{kg})$ & 0.141 & 0.063 & 2.25 & 0.03 \\
\hline & & Age (years) & 0.23 & 0.101 & 2.25 & 0.03 \\
\hline
\end{tabular}

${ }^{\star} \mathrm{MVC}=$ maximal voluntary quadriceps.

different in the two groups $(95 \%$ CI of difference 0.10 to $0.20, \mathrm{p}<0.001)$. Furthermore, controls activated their quadriceps significantly more than patients with OA $(95 \%$ CI of difference 11.51 to $31.34, p<0.001$ ) (fig 5). A proportion of controls and cases had more than full $(100 \%)$ activation, suggesting that the machine is overestimating.

CORRELATION/REGRESSION ANALYSIS

Increasing age was associated with a decline in proprioceptive acuity for both controls and patients $(r=0.30, \mathrm{p}<0.001)$. In subjects with knee OA increased postural sway was associated with increasing weight $(r=0.35, \mathrm{p}=0.007)$, reduced MVC $(r=-0.30, \mathrm{p}=0.025)$, and reduced MVC/body weight ( $r=-0.40, \mathrm{p}=0.002)$. Correlation was also observed with increasing disability $(r=0.34, \mathrm{p}=0.01)$ and knee pain $(r=0.30, \mathrm{p}=0.03)$.

Regression analysis showed that the presence of knee OA, obesity, and weak MVC were the most significant independent predictors of increased postural sway. The model accounted for $47 \%$ of variation in lateral postural sway (table 3A).

When subjects who had knee OA were considered separately, knee pain and the ratio of MVC/body weight were the most significant predictors of sway and the model accounted for $30 \%$ of the variation in sway (table $3 \mathrm{~B}$ ). The ratio of the two variables MVC and body weight was included in the analysis in addition to the variables themselves. This was because the effect of the interaction was thought to be important. In subjects with knee OA the ratio was a more significant predictor of postural lateral sway than either factor alone.

MANOVA analysis was performed on the 104 subjects (47 controls, 57 patients) for whom complete data were available. The analysis showed that controlling for weight, patients sway significantly more $(\mathrm{p}<0.001)$, have greater error in reproducing the criterion angle $(p<0.001)$, have less MVC ( $p<0.001)$, and less activation of the quadriceps $(p=0.002)$ than controls.

Exploring the determinants of physical function disability was not the prime objective of this study. However, from among the variables age, weight, postural sway, proprioception, MVC, quadriceps activation, and knee pain four predictors emerged. These were age, weight, MVC, and knee pain. The final model accounted for $66 \%$ of the variation in physical disability in subjects with knee $\mathrm{OA}$ of this cohort (table 3C).

\section{Discussion}

The main findings of this study are that subjects with knee OA, compared with age and sex matched controls, (a) sway more in both lateral and anteroposterior directions (under conditions of quiet standing position with eyes closed); (b) are less accurate in reproducing the criterion angle; (c) exert less force during voluntary isometric contraction of the quadriceps; and $(d)$ have less activation of the quadriceps muscle. These differences persisted after controlling for weight $(\mathrm{p}<0.001)$. The findings are in accord with previous studies that have investigated similar parameters in isolation or in limited combination employing similar techniques. $^{71620-23}$ This is the first study to assess all three parameters in the same patients with OA and in matched controls and to seek explanatory correlation between these and other constitutional factors, such as age, sex, and obesity.

The apparently healthy controls, although activating the quadriceps significantly more than subjects with knee OA, nevertheless failed to reach an average of maximal $(100 \%)$ activation reported in other studies employing the same methodology. ${ }^{16}$ Jones suggested a cut off point of $<77 \%$ to indicate failure of normal activation by an individual. ${ }^{16}$ Some controls in the present study, however, had $<70 \%$ activation, a finding reported in one previous study. ${ }^{24}$ There are several possible explanations for incomplete activation in subjects with apparently healthy knees. For example, it may well be that the variation in healthy subjects is wider than previously recorded. Alternatively, the position of the pads and the precise muscle fibres being tested may account for variation in the pattern of recruitment. Overall, however, a clear difference between the patients with OA and controls has been shown.

Quadriceps strength and proprioception are clearly important for balance control. The present study showed that both were compromised in subjects with knee OA. The observed increase in postural sway in these subjects may be due to impairment in both or one of these 
parameters. Interestingly, however, the correlation and regression analyses demonstrated an association between increased postural sway and reduced quadriceps strength but no clear association with reduced proprioception. The reason for this may be that proprioceptive input from ankle mechanoreceptors, muscle spindles, and the cutaneous receptors of the sole make an important contribution to the maintenance of static postural control, ${ }^{25}$ thus compensating for reduced knee proprioception during this assessment. ${ }^{26-29}$

Patients with $\mathrm{OA}$ and controls, although matched for age and sex, clearly differed in weight and in the ratio of quadriceps strength to weight. These differences, together with presence of knee pain, would seem to be important factors to explain the increased sway observed in subjects with OA. Indeed for subjects with knee OA, $30 \%$ of the variation in postural sway was attributable to the ratio of quadriceps strength to weight and level of pain.

It might therefore be that in OA muscle weakness resulting from the OA process, muscle dysfunction secondary to pain, and the additional effect of increased weight, all contribute to reduce the ability of muscle to maintain postural stability and thus lead to the observed increase in the frequency of sway. Factors such as obesity and quadriceps dysfunction, both for strength and proprioception, are potentially amenable to intervention. The present data would support the rationale for such interventions and the inclusion of such physiological assessments in prospective intervention trials.

This study also explored whether any of the measures undertaken in this study might be important determinants of physical disability. Of the factors examined knee pain, aging, increased weight, and reduced MVC emerged as significant predictors of disability, the final model accounting for $66 \%$ of the variation observed. This is consistent with findings of other investigators. ${ }^{30-32} \mathrm{We}$ also sought predictors of impaired proprioceptive acuity and found increasing age to be the common factor for both patients with OA and controls. This finding accords with previous reports ${ }^{15}{ }^{33-35}$ and suggests that a decline in proprioceptive acuity is a normal accompaniment of aging.

There are caveats to this study. Firstly, the patients with OA were derived from a hospital setting and their symptoms and disability are likely to be representative of the more severe end of the OA population. Controls were selected to be free from knee pain and overt balance disturbance. Such selection bias in both groups may limit the generalisability of the data. Secondly, non-OA status for controls was based on a negative history of pain and normal examination findings alone. Some of these subjects might have had radiographic changes of OA or other occult knee pathology. However, radiographic changes of $\mathrm{OA}$ in asymptomatic subjects in this age group would not be expected to be high. In a community based study undertaken by our group, ${ }^{36} 7.5 \%$ of asymptomatic subjects had grade 2 osteophytes and $11.3 \%$ grade 2 joint space narrowing of any compartment. It is likely, therefore, that the majority of our controls had structurally normal knees and that differences seen between the two groups predominantly relate to the OA process. A more important caveat to our study, however, is that all OA subjects had knee pain. We were therefore unable to assess separately the individual contributions of pain and structural change to the impaired strength, reduced proprioception, and increased sway that we observed in the patient group. Future studies that include subjects with and without pain, with and without structural change, are required to consider this question more fully. Our method of assessment for balance was also rather limited, and future studies that investigate the role of proprioception of the ankle joint and the strength of calf muscles in the maintenance of static balance in subjects with knee OA would seem warranted.

We wish to thank all those who volunteered to participate in the study; Joyce Stevenson for statistical advice; and the Ministry of Higher Education, Sultanate of Oman for the research scholarship for $\mathrm{BH}$.

1 Assimakopoulos A, Katonis P, Agapitos M. The innervation of the human meniscus. Clin Orthop 1992;275:232-6.

2 Assimakopoulos AP, Agapitos EV, Exarchou EI. A histological study of the innervation of human lateral collateral ligament. Knee 1995;2:163-4

3 Dell Valle ME, Harwin SF, Maestro A, Murcia A, Vega JA. Immunohistochemical analysis of mechanoreceptors in human posterior cruciate ligament: a demonstration of its proprioceptive role and clinical relevance. J Arthroplasty 1998;13:916-22.

4 Schutte MJ, Dabezies EJ, Zimny ML. Neural anatomy of human anterior cruciate ligament. J Bone Joint Surg Am 1987;69:243-7.

5 Brandt KD. The pathogenesis of osteoarthritis. Rheumatology Reviews 1991;1:3-11.

6 Martel-Pelletier J. Pathophysiology of osteoarthritis. OsteoMartel-Pelletier J. Pathophysiology

7 Barrett DS, Cobb AG, Bentley G. Joint proprioception in normal, osteoarthritic and replaced knees. J Bone Joint Surg Br 1991;73:53-6.

8 Marks R, Quinney HA, Wessel J. Proprioceptive sensibility in women with normal and osteoarthritic knee joints. Clin Rheumatol 1993;12:170-5.

9 Nordesjo Lars-Olof, Nordgren B, Wigren A. Isometric strength and endurance in patients with severe rheumatoid arthritis or osteoarthritis in the knee joint. Scand J Rheumatol 1983;12:152-6.

10 Hurley MV, Newham DJ. The influence of arthrogenous muscle inhibition on quadriceps rehabilitation of patients with early, unilateral osteoarthritic knees. Br J Rheumatol 1993;32:127-31.

11 Brucini M, Duranti R, Galletti R. Pain thresholds and electromyographic features of periarticular muscles in patients
with osteoarthritis of the knee. Pain 1981;10:57-66.

12 Patla A, Frank J, Winter D. Assessment of balance control in the elderly: major issues. Physiotherapy Canada 1990;42: 89-97.

13 Winter DA, Patla A, Frank J. Assessment of balance control in humans. Med Prog Technol 1990;16:31-51

14 Bellamy N, Buchanan WW, Goldsmith CH, Campbell J, Stitt LW. Validation study of WOMAC: a health status instrument for measuring clinically important patient relevant outcomes to antirheumatic drug therapy in patients with osteoarthritis of the hip and knee. J Rheumatol 1988;15:1833-40.

15 Skinner HB, Barrack RL, Cook SD. Age-related decline in proprioception. Clin Orthop 1984:208-11.

16 Jones AC. Assessment and muscle function in knee osteoarthritis. Nottingham, 1994. (DM thesis.)

17 Bigland-Ritchie B, Ferbush F, Woods JJ. Neuromuscular transmission and muscle activation in human post-fatigue ischemia. J Physiol 1986;365:76P.

18 Sutton A, Muir K, Jones A. Two knees or one person: data analysis strategies for paired joints or organs. Ann Rheum Dis 1997;56:401-2.

19 Bland JM, Altman DG. Statistical methods for assessing agreement between two methods of clinical measurement. Lancet 1986;i:307-10.

20 Hurley MV, Scott DL, Newham DJ. Sensorimotor changes and functional performance in patients with knee osteoarthritis. Ann Rheum Dis 1997;56:641-8.

21 O'Reilly S, Jones A, Doherty M. Quadriceps weakness in knee osteoarthritis: the effect on pain and disability. Ann Rheum Dis 1998;57:588-94.

22 Skinner HB, Barrack RL. Joint position sense in the normal and pathologic knee joint. J Electromyogr Kinesiol 1991;1: 180-90. 
23 Wegener L, Kisner C, Nichols D. Static and dynamic balener L, Kisner C, Nichols D. Static and dynamic balance responses in persons with bilateral knee

24 Rutherford OM, Jones DA, Newham DJ. Clinical and experimental application of the percutaneous twitch superimposition technique for the study of human muscle activation. J Neurol Neurosurg Psychiatry 1986;49:1288-91.

25 Henry SM, Fung J, Horak FB. EMG responses to maintain stance during multidirectional surface translations. J Neurophysiol 1998;80:1939-50.

26 Holme E, Magnussen SP, Becher K, Aagaard P. The effect of supervised rehabilitation on strength, postural sway, position sense and re-injury risk after acute ankle ligamen sprain. Scand J Med Sci Sport 1999;9:104-9.

27 Kavounoudias A, Gilhodes J, Régine R. From balance regulation to body orientation: two goals for muscle proprioceptive information processing? Exp Brain Res 1999; 124:80-8.

28 Maki BE, Perrt SD, McIlroy WE. Effect of facilitation of sensation from plantar foot-surface boundaries on postural sensation from plantar foot-surface boundaries on postural Sci Med Sci 1999;54:M281-7.

29 Szturm T, Fallang B. Effects of varying acceleration of platform translation and toe-up rotations on the pattern and form translation and toe-up rotations on the pattern and
magnitude of balance reactions in humans. J Vestib Res magnitude of bala
30 Davis MA, Ettinger WH, Neuhaus JM. Knee osteoarthritis and physical functioning: Evidence from the NHANES epidemiologic follow up study. J Rheumatol 1991;18: $591-8$

31 McAlindon TE, Cooper C, Kirwan JR, Dieppe PA. Knee pain and disability in the community. $\mathrm{Br} \mathrm{J}$ Rheumatol 1992;31:189-92.

32 McAlindon TE, Cooper C, Kirwan JA, Dieppe P. Determinants of disability in osteoarthritis of the knee. Ann Rheum Dis 1993;52:258-62.

33 Hurley MV, Rees J, Newham DJ. Quadriceps function, proprioceptive acuity and functional performance in healthy young, middle-aged and elderly subjects. Age Ageing 1998; 27:55-62.

34 Kaplan FS, Nixon JE, Reitz M. Age related changes in proprioception and sensation of joint position. Acta Orthop Scand 1985;56:72-4.

35 Petrella RJ, Lattanzio PJ, Nelson MG. Effects of age and activity on knee joint proprioception. Am J Phys Med Rehabil 1997;76:235-41.

36 Lanyon P, O'Reilly S, Jones A, Doherty M. Radiographic assessment of symptomatic knee osteoarthritis in the community: definitions and normal joint space. Ann Rheum Dis 1998;57:595-601.

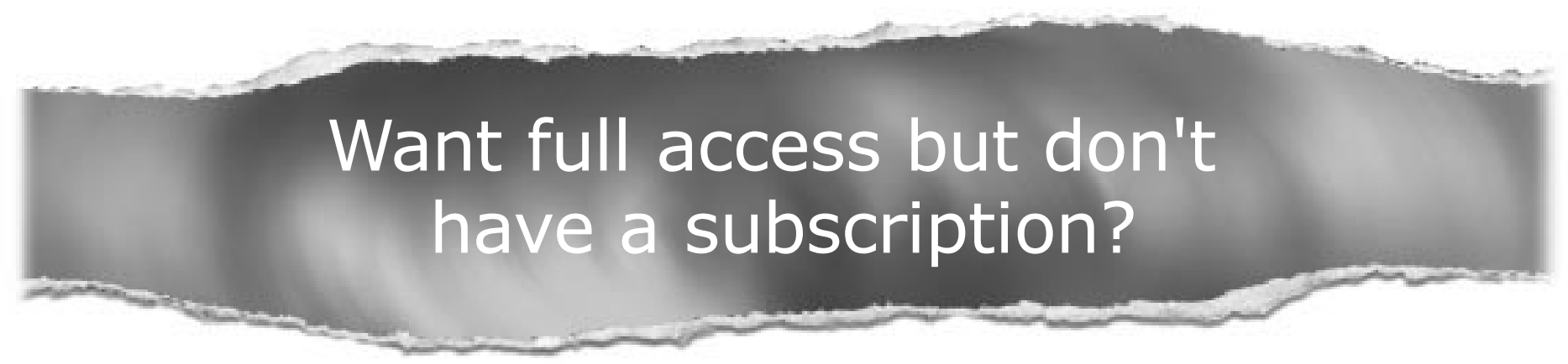

\section{Pay per access}

For just US $\$ 25$ you can have instant access to the whole website for 30 days. During this time you will be able to access the full text for all issues (including supplements) available. You will also be able to download and print any relevant pdf files for personal use, and take advantage of all the special features Annals of the Rheumatic Diseases online has to offer.

www.annrheumdis.com 